\title{
Session 5: Antibody repertoire
}

\author{
Tuesday 30th October 2007. Moderator: To be confirmed
}

[09.00-09.45]

[Keynote Lecture]

Human B cell development: Immunization with influenza virus results in a rapid and profoundly clonal B cell response

J. Donald Capra, Ken Smith, Patrick Wilson

Oklahoma Medical Research Foundation, USA; and

Jens Wrammert and Rafi Ahmed, Emory University

Abstract: Human peripheral B cells can be divided into several subsets based on there developmental stage. Over the years our laboratory has characterized these subsets and tracked B cells from mature naïve to their end stages: memory cells and plasmablasts. In the past, studying tonsilar germinal center cells we have described a number of novel mechanisms of antibody diversity. Over the last year we have turned our attention to the impact of standard influenza vaccination on B cell subsets. Surprisingly, seven days after immunization, a burst of plasmablasts appears in the peripheral blood. These cells are not there on day 5 and are gone by day 9 . The plamablasts were isolated and over $75 \%$ were found to be secreting IgG anti influenza antibodies. Structural studies on these antibodies revealed them to be clonal in that in any one person only three or four major clones dominated the entire immune response. Human monoclonal antibodies were made by recombinant techniques from single cell PCR analysis of the plamablasts. These antibodies had an extremely high affinity for influenza virus (especially of the previous year's strain) and were highly specific for strain variation in the influenza virus. In addition to providing new insights into the secondary immune response, this approach is a simple way to produce human monoclonal antibodies.

[09.45-10.05]

Lessons learnt from nature. Immunoglobulin repertoire analysis and single chain $F v$ antibody

\author{
libraries from B cells in breast carcinomas and \\ melanomas \\ Beatrix Kotlan $^{\mathrm{a}, \mathrm{b}, \mathrm{d}}$, Michael McKnight ${ }^{\mathrm{b}}$, Andrea \\ Ladanyi $^{\mathrm{c}}$, Mepur H Ravindranath ${ }^{\mathrm{d}}$, Erzsebet Raso ${ }^{\mathrm{c}}$, \\ Jozsef Toth ${ }^{\mathrm{c}}$, Jozsef Timar ${ }^{\mathrm{c}}$ and Mark C Glassy ${ }^{\mathrm{b}}$ \\ ${ }^{a}$ National Medical Center, Szabolcs street 33-35, \\ Budapest, H-1135, Hungary \\ ${ }^{\mathrm{b}}$ Rajko Medenica Research Foundation, 11211 \\ Sorrento Valley Rd, San Diego, 92121, CA \\ ${ }^{\mathrm{c}}$ National Institute of Oncology, Rath Gy street 7-9, \\ Budapest, H-1122 Hungary \\ d John Wayne Cancer Institute, 2200 Santa Monica \\ Blvd, Santa Monica, 90404, CA, USA
}

E.mail: KotlanB@netscape.net

Key words: immunoglobuline, B cells, breast

carcinomas, ganglioside, melanoma

Introduction: Human monoclonal antibodies against tumor-associated ganglioside antigens have the potential for tumor therapeutic reagents. We report here a novel strategy to obtain such antibodies. B cells infiltrating breast carcinomas and melanomas (TIL-B) were investigated through detailed immunoglobuline repertoire analysis and antibody fragment phage display libraries in terms of tumor antigen specific binding potential. We questioned the characteristics of these different tumor types.

Description of the work: Different types of breast carcinoma and melanoma tumor tissues were processed for further cellular and molecular studies. Heavy (VH) and light chain $(\mathrm{V} \kappa, \mathrm{V} \lambda)$ immunoglobulin variable region genes were amplified. Single chain Fv ( $\mathrm{scFv}$ ) antibody fragments were constructed and phage display libraries generated. We compared our previously developed method to other techniques. The effectiveness of the selection process in respect of tumor associated ganglioside specific antibody fragments was tested against various tumor cell membranes. Selected tumor binder antibody fragments of breast cancer and melanoma TIL-B libraries were investigated in cell 
ELISA, immunofluorescence FACS analysis, confocal microscopy. DNA sequence analysis and biochemical investigations were done to define the targeted tumor associated antigens.

Summary of results: Heavy and light chain immunoglobulin variable region genes could be amplified from any of the investigated tissues. Cloned $\mathrm{VH}$ and $\mathrm{V} \kappa$ gene regions were sequenced and subject of a detailed comparative DNA sequence analysis. From the single chain Fv antibody fragment phage libraries generated specific tumor binder antibody fragments could be selected. Main characteristics of TIL-B immunoglobulin repertoires of different tumors could be stated, and major target antigens were defined also.

Conclusion: Our results suggest that B cells accumulated in breast carcinomas or melanomas have the potential to reveal key tumor associated antigens.

Acknowledgements: Supporting grants to B. Kotlan (Fulbright Research Scholarship 1206103 (26.0911), OTKA T048933, OTKA T030380, NATO CLG 978639, Rajko Medenica Research Foundation).

[10.05-10.25]

\author{
The molecular basis of autoreactive primary \\ immunoglobulins \\ Gary R McLean ${ }^{\mathrm{a}, \mathrm{b}}$, Chantal Cho ${ }^{\mathrm{a}}$ and John W \\ Schrader $^{\mathrm{a}}$ \\ ${ }^{\mathrm{a}}$ The Biomedical Research Centre, Vancouver, BC, \\ Canada \\ ${ }^{\mathrm{b}}$ The University of Texas Medical School, Houston, \\ TX, USA
}

The stochastic nature of the antibody variable (V) region recombination process ensures a diverse repertoire of binding specificities but it also generates immunoglobulins that react with self and thus the threat of autoimmune disease. While the majority of antibodies expressed by early immature human B cells are selfreactive, most of these reactivities are removed from the repertoire in a stepwise process in the bone marrow and in the transition to mature B cells in the periphery. Our recent work investigating the human antibody response to the AD-2 epitope of glycoprotein $\mathrm{B}(\mathrm{gB})$ of human cytomegalovirus (HCMV) showed that it is dominated by a family of closely related somatically mutated antibodies. These antibodies potently neutralize viral infectivity and the genes encoding them are derived from two commonly used germ-line $\mathrm{V}$ region genes, IGHV3-30 and IGKV3-11. Recombination of these $\mathrm{V}$ genes with the appropriate junctional diversity generates genes that encode primary immunoglobulins that bind to AD-2. To further understand the initial primary immunoglobulin response to AD-2S1 we synthesized the germ-line-based ancestor of this family of antibodies and showed that it bound $\mathrm{gB}$ at the $\mathrm{AD}-2$ epitope. The germ-line ancestor of a second family of antibodies likewise binds to gB. Interestingly, we found that one of the ancestral primary immunoglobulins, but not the other, also recognized autoantigens. In contrast, the hypermutated derivatives did not demonstrate autoreactivity and minor structural changes in the primary immunoglobulin were sufficient to generate or abolish autoreactivity or to change specificity. This demonstration that the ancestor of a highly mutated, nonautoreactive antiviral $\mathrm{IgG}$ antibody binds nuclear and cell-surface autoantigens indicates that self-reactivity is not necessarily a barrier to development into a follicular B lymphocyte that undergoes antigen-initiated affinity maturation.

\section{[10.25-10.45]}

\section{Identification and characterization of cynomolgus immunoglobulin constant regions}

Taruna Arora

Amgen Inc: Primates, such as cynomolgus monkeys (Macaca fasicularis), can be used as animal models for pharmacokinetics and toxicology studies. Antibodies may be introduced into the monkeys to evaluate toxic effects against a given biological target. However, a foreign antibody may cause an immune response in the monkey that inhibits its function and interferes with this evaluation. In addition, interactions between human Fc and cyno Fc gamma receptors are not understood. The use of chimeric antibodies composed of human variable regions directed at the disease target and monkey constant regions might reduce the immunogenic response and allow for more relevant $\mathrm{Fc}-\mathrm{FcR}$ interactions. We have cloned cynomolgus monkey immunoglobulin constant regions and used them to construct chimeric antibodies. In vitro testing has demonstrated that the heavy chain constant regions differ in their Fc binding and effector functions. 